\title{
THE INTERACTION OF AN ACOUSTIC WAVE AND AN ELASTIC SPHERICAL SHELL*
}

\author{
BY \\ P. MANN-NACHBAR \\ Missile Systems Division, Lockheed Aircraft Corp.
}

\begin{abstract}
The effect of the impact of a plane pressure wave on an elastic spherical shell is considered, taking into account both the incident and diffracted waves. An infinite series (mode) solution is used and numerical results are obtained for the case of a steel shell in water. It is shown that a simple quasi-static system can be used to find a very good approximation for the stress. The total acceleration on initial impact is found exactly and seen to differ markedly from the accelerations associated with the lower vibrational modes.
\end{abstract}

1. Introduction. This problem was suggested by G. F. Carrier in consequence of work previously done by him on a related problem [1] ${ }^{1}$ (see also [2]).

An attempt had been made to determine the response of a cylindrical shell to an acoustic wave. The form of the functions dealt with in the analysis made it difficult to obtain accurate explicit results. If the obstacle is taken to be spherical in shape, we still have a practical though highly simplified model of an actual physical structure; moreover, the problem becomes mathematically simpler, admitting of exact solutions for the deformation and accompanying strains in the elastic body. It was therefore decided to treat the case of the sphere in detail.

2. Forced vibrations of a thin spherical shell. Consider a closed shell of thickness $h$ with $h \ll R$ where $R$ is the radius of the middle surface. The motion of any closed oval $^{2}$ shell, in particular a spherical shell is, by a theorem of Jellett [3], primarily extensional. Therefore, the general membrane theory of shells is applicable. If we locate the origin of our coordinate system at the center of the shell and choose as the $z$-axis the direction of propagation of the incoming wave, then we have the additional simplification of symmetrical loading (see Fig. 1A).

The equations of dynamic equilibrium for an element of shell may, therefore, be written [4],

$$
\begin{aligned}
\frac{\partial}{\partial \theta}\left(N_{\theta} \sin \theta\right)-N_{\phi} \cos \theta-\rho h \frac{\partial^{2} v}{\partial t^{2}} R \sin \theta & =0, \\
N_{\theta}+N_{\phi}-\left(s-\rho h \frac{\partial^{2} w}{\partial t^{2}}\right) R & =0 .
\end{aligned}
$$

Here $N_{\phi}$ and $N_{\theta}$ are the normal forces/unit length acting on the sides of the element (see Fig. 1B), $s$ is the external load (radial in direction), $\rho$ is the shell density, and $v$ and $w$ are the tangential and radial components of the displacement.

$N_{\phi}$ and $N_{\theta}$ can be eliminated from the Eqs. (2.1) and (2.2) by the use of Hooke's

*Received February 10, 1956. The results in this paper appeared previously as Brown University report to the Office of Naval Research under Contract N7onr-35810, No. B11-11/34, 1953.

${ }^{1}$ Numbers in brackets refer to the Bibliography at the end of the paper.

'Principal radii of curvature finite and of the same sign. 


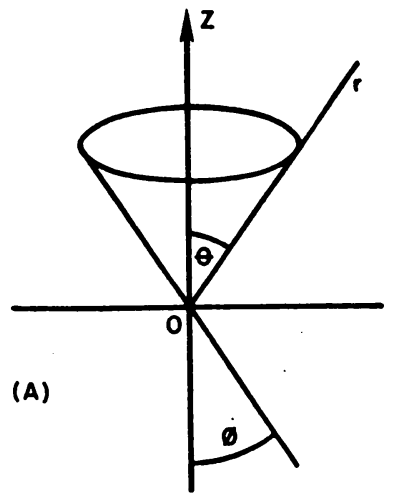

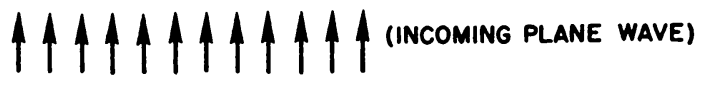

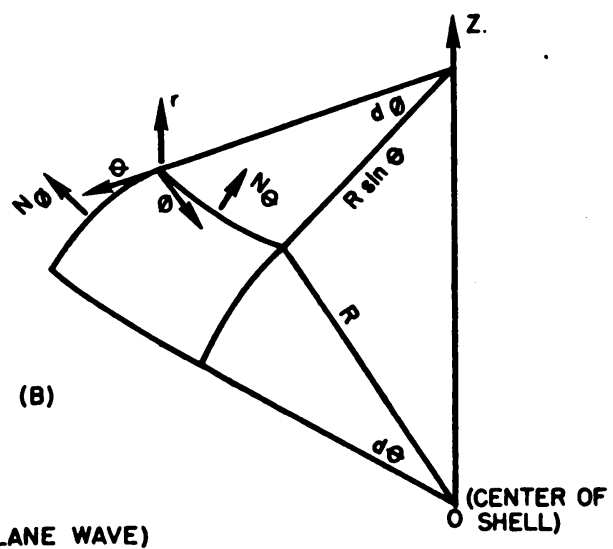

Fra. 1. Geometry of the problem.

law and the expressions for the strains in spherical coordinates. The resulting equations can be further simplified by the introduction of the dimensionless variables ( $E$ is Young's modulus),

$$
w^{*} \equiv w / R ; \quad v^{*} \equiv v / R ; \quad \tau^{2} \equiv t^{2} / t_{0}^{2} \equiv E t^{2} /\left(R^{2} \rho\right) ; \quad s^{*} \equiv s / s_{0} \equiv R s /(E h) .
$$

After some manipulation, we obtain for the equations of motion the following relations in $w^{*}$ and $v^{*}$ only ( $\nu$ is the Poisson's ratio).

$$
\begin{aligned}
& M\left(v^{*}\right)=2(1-\left.\nu^{2}\right) \frac{\partial^{2} v^{*}}{\partial \tau^{2}}+\left(1-\nu^{2}\right)(1-\nu) \frac{\partial^{4} v^{*}}{\partial \tau^{4}}+2 v v^{*}+\nu(1-\nu) \frac{\partial^{2} v^{*}}{\partial \tau^{2}} \\
&-2 \frac{\partial^{2} v^{*}}{\partial \theta^{2}}-(1-\nu) \frac{\partial^{4} v^{*}}{\partial \tau^{2} \partial \theta^{2}}+2 v^{*} \cot ^{2} \theta+(1-\nu)\left(\cot ^{2} \theta\right) \frac{\partial^{2} v^{*}}{\partial \tau^{2}} \\
&-2(\cot \theta) \frac{\partial v^{*}}{\partial \theta}-(1-\nu)(\cot \theta) \frac{\partial^{3} v^{*}}{\partial \theta \partial \tau^{2}}+(1+\nu)\left[(\cot \theta) \frac{\partial v^{*}}{\partial \theta}\right. \\
&\left.-v^{*} \csc ^{2} \theta+\frac{\partial^{2} v^{*}}{\partial \theta^{2}}\right]=\left(1-\nu^{2}\right) \frac{\partial s^{*}}{\partial \theta} \\
& L\left(w^{*}\right)=(\cot \theta)\left(\frac{\partial w^{*}}{\partial \theta}+\frac{\partial^{3} w^{*}}{\partial \tau^{2} \partial \theta}\right)+2 w^{*}+\frac{\partial^{2} w^{*}}{\partial \theta^{2}}+\frac{\partial^{4} w^{*}}{\partial \tau^{2} \frac{\partial}{\partial \theta^{2}}} \\
&-\left(1-\nu^{2}\right) \frac{\partial^{4} w^{*}}{\partial \tau^{4}}+(1-\nu) \frac{\partial^{2} w^{*}}{\partial \tau^{2}}-2(1+\nu) \frac{\partial^{2} w^{*}}{\partial \tau^{2}} \\
&=\cot \theta \frac{\partial s^{*}}{\partial \theta}-\left(1-\nu^{2}\right) \frac{\partial^{2} s^{*}}{\partial \tau^{2}}+\frac{\partial^{2} s^{*}}{\partial \theta^{2}}+(1-\nu) s^{*} .
\end{aligned}
$$

3. Acoustic wave propagation. The linearized theory of wave propagation yields the acoustic wave equation

$$
\Delta \phi-\lambda^{2} \phi_{. r \tau}=0,
$$

where $\phi$ is the velocity potential, $\Delta \phi$ is the Laplacian in spherical coordinates, $\lambda^{2}=$ 
$R^{2} /\left(t_{0}^{2} c^{2}\right)=E /\left(\rho c^{2}\right)$, and $c$ is the acoustic speed of the fluid. The pressure perturbation $P$ is given by

$$
\begin{gathered}
P=-p^{*} \phi_{. \tau}(r, \theta, \tau), \\
p^{*}=\rho_{f} R^{2} / t_{0}^{2}=\rho_{f} E / \rho,
\end{gathered}
$$

where $\rho_{f}$ is the fluid density. The applied stress, $s$, of (2.2) must, of course, be the same as the acoustic pressure $P$ at the surface of the sphere. We have, in fact

$$
\begin{aligned}
s^{*}=-P / s_{0} & =\left(p^{*} / s_{0}\right) \phi_{. \tau}(1, \theta, \tau)=\left(\rho_{f} R / \rho h\right) \phi_{. \tau}(1, \theta, \tau) \\
& \equiv \beta \phi_{. \tau}(1, \theta, \tau) .
\end{aligned}
$$

4. The interaction problem. We now pose the following problem. An incoming plane wave, $\phi_{0}$, obeying (3.1) impinges on an elastic spherical shell causing it to vibrate according to (2.3) and (2.4). The vibrating body acts as a scatterer and, to a lesser extent, as a radiator. The outgoing waves (scattered and radiated) also obeying (3.1), in turn influence the nature of the vibrations. At the surface of the shell, the radial velocity of the fluid, $\phi, r(1, \theta, \tau)$ must be equal to the radial velocity of the shell, $\omega_{. r}^{*}(\theta, \tau)$. We wish to determine the motion of the sphere and the pressure distribution associated with the incoming and outgoing waves.

The pressure associated with the incident wave is taken to be [1]

$$
P= \begin{cases}Q_{0} \exp [\delta(z-\tau / \lambda)] & z \leq \tau / \lambda \\ 0 & z>\tau / \lambda\end{cases}
$$

so that the initial velocity potential is

$$
\overrightarrow{\phi_{0}}= \begin{cases}\left(Q_{0} \lambda / p^{*} \delta\right) \exp [\delta(z-\tau / \lambda)]-\left(Q_{0} \lambda / p^{*} \delta\right) & z \leq \tau / \lambda \\ 0 & z>\tau / \lambda .\end{cases}
$$

For simplicity let $\psi_{0}, \psi, x, W$ and $V$ be defined as:

$$
\begin{array}{rlrl}
\phi_{0} & \equiv\left(Q_{0} / p^{*}\right) \psi_{0}, & \phi \equiv\left(Q_{0} / p^{*}\right)\left(\psi_{0}+\psi\right) \equiv\left(Q_{0} / p^{*}\right) \chi, \\
w^{*} \equiv\left(Q_{0} / p^{*}\right) W, & v^{*} \equiv\left(Q_{0} / p^{*}\right) V .
\end{array}
$$

Then our boundary value problem is defined by the following set of equations ${ }^{3}$.

$$
\begin{aligned}
& L(W)=\beta\left[\chi_{,{ }_{\text {or }}}(1, \theta, \tau) \cot \theta-\left(1-\nu^{2}\right) \chi_{. \mathrm{rrr}}(1, \theta, \tau)\right. \\
& +\chi_{. \theta \theta \tau}(1, \theta, \tau)+(1-\nu) \chi_{. r}(1, \theta, \tau), \\
& M(V)=\beta\left(1-\nu^{2}\right) \chi, \theta_{\tau}(1, \theta, \tau) \text {, } \\
& \Delta \psi-\lambda^{2} \psi_{. r}=0, \\
& W_{.,}(\theta, \tau)=\chi_{. r}(1, \theta, \tau), \quad \text { [boundary condition] } \\
& \psi_{0}= \begin{cases}(\lambda / \delta) \exp [\delta(z-\tau / \lambda)]-\lambda / \delta & z \leq \tau / \lambda \\
0 & z>\tau / \lambda .\end{cases}
\end{aligned}
$$

The operators $L$ and $M$ which appear here are those given in (2.3) and (2.4). 
These equations will be more easily handled if Fourier transforms are first introduced to eliminate the time dependence. Denote the transform of a function $F$ by $F^{\boldsymbol{T}}$. Then $F$ and $F^{T}$ are related by

$$
\begin{gathered}
F^{r}(r, \theta, \xi)=\int_{-\infty}^{\infty} F(r, \theta, \tau) \exp (-i \xi \tau) d \tau, \\
I m \xi=-i \alpha, \quad \alpha>0, \\
F(r, \theta, \tau)=\frac{1}{2 \pi} \int_{-i \alpha+\infty}^{-i \alpha-\infty} F^{\tau}(r, \theta, \xi) \exp (i \xi \tau) d \xi .
\end{gathered}
$$

Applying (4.9a) to (4.4) through (4.8) we obtain

$$
L^{T}\left(W^{T}\right)=i \beta \xi\left[\chi_{, \theta}^{T} \cot \theta+\chi_{\cdot \theta \theta}^{T}(1, \theta, \xi)+(1-\nu) \chi^{T}+\xi^{2}\left(1-\nu^{2}\right) \chi^{T}\right],
$$

where

$$
\begin{gathered}
L^{T}=(\cot \theta)\left(\frac{\partial}{\partial \theta}-\xi^{2} \frac{\partial}{\partial \theta}\right)+2+\frac{\partial^{2}}{\partial \theta^{2}}-\xi^{2} \frac{\partial^{2}}{\partial \theta^{2}}-\left(1-\nu^{2}\right) \xi^{4} \\
-(1-\nu) \xi^{2}+2(1+\nu) \xi^{2}, \\
M^{T}\left(V^{T}\right)=i \beta \xi\left(1-\nu^{2}\right) \chi_{0}^{T},
\end{gathered}
$$

where

$$
\begin{gathered}
M^{T}=-2\left(1-\nu^{2}\right) \xi^{2}+\left(1-\nu^{2}\right)(1-\nu) \xi^{4}+2 \nu-\nu(1-\nu) \xi^{2} \\
-2 \frac{\partial^{2}}{\partial \theta^{2}}+(1-\nu) \xi^{2}+2 \cot ^{2} \theta-(1-\nu) \xi^{2} \cot ^{2} \theta \\
-2(\cot \theta) \frac{\partial}{\partial \theta}+(1-\nu) \xi^{2}(\cot \theta) \frac{\partial}{\partial \theta}+(1+\nu)\left[(\cot \theta) \frac{\partial}{\partial \theta}-\csc ^{2} \theta+\frac{\partial^{2}}{\partial \theta^{2}}\right] \\
\Delta \psi^{T}+\lambda^{2} \xi^{2} \psi^{T}=0, \\
i \xi W^{T}(\theta, \xi)=\chi_{.,}^{T}(1, \theta, \xi), \\
\psi_{0}^{T}=-\frac{\exp (-i z \lambda \xi)}{i \xi[(\delta / \lambda)+i \xi]}=-f(\xi) \exp (-i \lambda r \xi \cos \theta) ; \\
f(\xi) \equiv\{i \xi[(\delta / \lambda)+i \xi]\}^{-1} .
\end{gathered}
$$

It can be shown, using the method of separation of variables on (4.12), that $\psi^{T}$ is of the form

$$
r^{-\frac{1}{2}}\left[J_{n+\xi}(\lambda \xi r)+C Y_{n+\frac{1}{3}}(\lambda \xi r)\right] P_{n}(\cos \theta) .
$$

Since the waves associated with $\psi^{T}$ must be outwardly moving, take $C=-i$ and write:

$$
\psi^{T}=\sum_{n=0}^{\infty} A_{n}^{T}(\xi) h_{n}^{(2)}(\lambda \xi r) P_{n}(\cos \theta),
$$

where

$$
h_{n}^{(2)}(\lambda \xi r)=(\pi / 2 \lambda \xi r)^{\frac{1}{3}}\left[J_{n+\xi}(\lambda \xi r)-i Y_{n+\xi}(\lambda \xi r)\right] .
$$


$\psi_{0}^{T}$ may be expanded similarly

$$
\begin{aligned}
\psi_{0}^{T}(r, \theta, \xi) & =-f(\xi) \sum_{n=0}^{\infty}(2 n+1)(-i)^{n} j_{n}(\lambda \xi r) P_{n}(\cos \theta) \\
& \equiv \sum_{n=0}^{\infty} B_{n}(\xi) j_{n}(\lambda \xi r) P_{n}(\cos \theta)
\end{aligned}
$$

where

$$
j_{n}(\lambda \xi r)=(\pi / 2 \lambda \xi r)^{\xi} J_{n+1}(\lambda \xi r) .
$$

From the work of Lamb [5], we know that the complete solutions for $W^{T}$ and $V^{T}$ may be written:

$$
\begin{gathered}
W^{T}(\theta, \xi)=\sum_{n=0}^{\infty} W_{n}^{T}(\xi) P_{n}(\cos \theta) \\
V^{x}(\theta, \xi)=-\sum_{n=0}^{\infty} V_{n}^{T}(\xi) P_{n}^{1}(\cos \theta)=-\sum_{n=0}^{\infty} V_{n}^{T} P_{n}^{\prime}(\cos \theta) \sin \theta
\end{gathered}
$$

Substituting these expressions into (4.10), (4.11) and (4.13), we get three algebraic equations for the three unknowns $W_{n}^{T}, V_{n}^{T}$ and $A_{n}^{T}$, which are readily solved to give:

$$
\begin{aligned}
W_{n}^{T} & =\frac{(2 n+1)(-i)^{n} C_{2}}{\lambda \xi(i \xi)^{2}[(\delta / \lambda)+i \xi]\left[C_{1} \lambda h_{n}^{(2) \prime}+i C_{2} h_{n}^{(2)}\right]}, \\
V_{n}^{T} & =\frac{-(2 n+1)(-i)^{n}(1+\nu) \beta}{\lambda \xi(i \xi)[(\delta / \lambda)+i \xi]\left[C_{1} \lambda h_{n}^{(2) \prime}+i C_{2} h_{n}^{(2)}\right]}, \\
A_{n}^{T} & =-\frac{1}{2}\left[1+\frac{C_{1} \lambda h_{n}^{(1) \prime}+i C_{1} h_{n}^{(1)}}{C_{1} \lambda h_{n}^{(2) \prime}+i C_{2} h_{n}^{(2)}}\right] \frac{1}{\xi^{2}}(2 n+1)(-i)^{n}
\end{aligned}
$$

where

$$
\begin{aligned}
& C_{1}=\left(1-\xi^{2}\right)\left(n^{2}+n\right)-2+\left(1-\nu^{2}\right) \xi^{4}+(1-\nu) \xi^{2}-2(1+\nu) \xi^{2} \\
& C_{2}=i \beta \xi\left[\left(1-\nu^{2}\right) \xi^{2}-\left(n^{2}+n\right)+(1-\nu)\right]
\end{aligned}
$$

The quantities of physical interest are the stresses, radial acceleration and the total pressure distribution. These can now all be found, at least in principle, from (4.19), (4.20), (4.21) and the inversion formula (4.9b).

5. Numerical results for the shell. For each vibrational mode, the transforms of the stress components will be linear combinations of $W_{n}^{T}$ and $V_{n}^{T}$; e.g. for the $n$th mode, $N_{\phi}^{T}$ will be given by

$$
\left[Q_{0} R /\left(1-\nu^{2}\right) \beta\right]\left[(1+\nu) W_{n}^{T} P_{n}(\cos \theta)-V_{n}^{T} P_{n}^{1}(\cos \theta) \cot \theta-\nu V_{n}^{T} \frac{\partial P_{n}^{1}(\cos \theta)}{\partial \theta}\right] .
$$

The transform of the total radial acceleration, $\partial^{2} w / \partial \tau^{2}$, will be $-\left(Q_{0} R^{2} / E h \beta\right) \xi^{2} W^{T}$ or, for the $n$th mode only

$$
-\left(Q_{0} R^{2} / E h \beta\right) \xi^{2} W_{n}^{\tau} P_{n}(\cos \theta) .
$$

From (4.19) and (4.20) we see that these expressions are regular in $\xi$ except for a finite number of poles. The theorem of residues and Jordan's lemma can therefore be used to evaluate the integral of $(4.9 \mathrm{~b})$. The computation was carried out in detail for the three 
lowest modes, and the results are shown for the point $\theta=\pi$ in Figs. 2 and 3. $N_{\phi}$ only is shown in Fig. 2 since the two tangential stress components are equal for $\theta=\pi$.

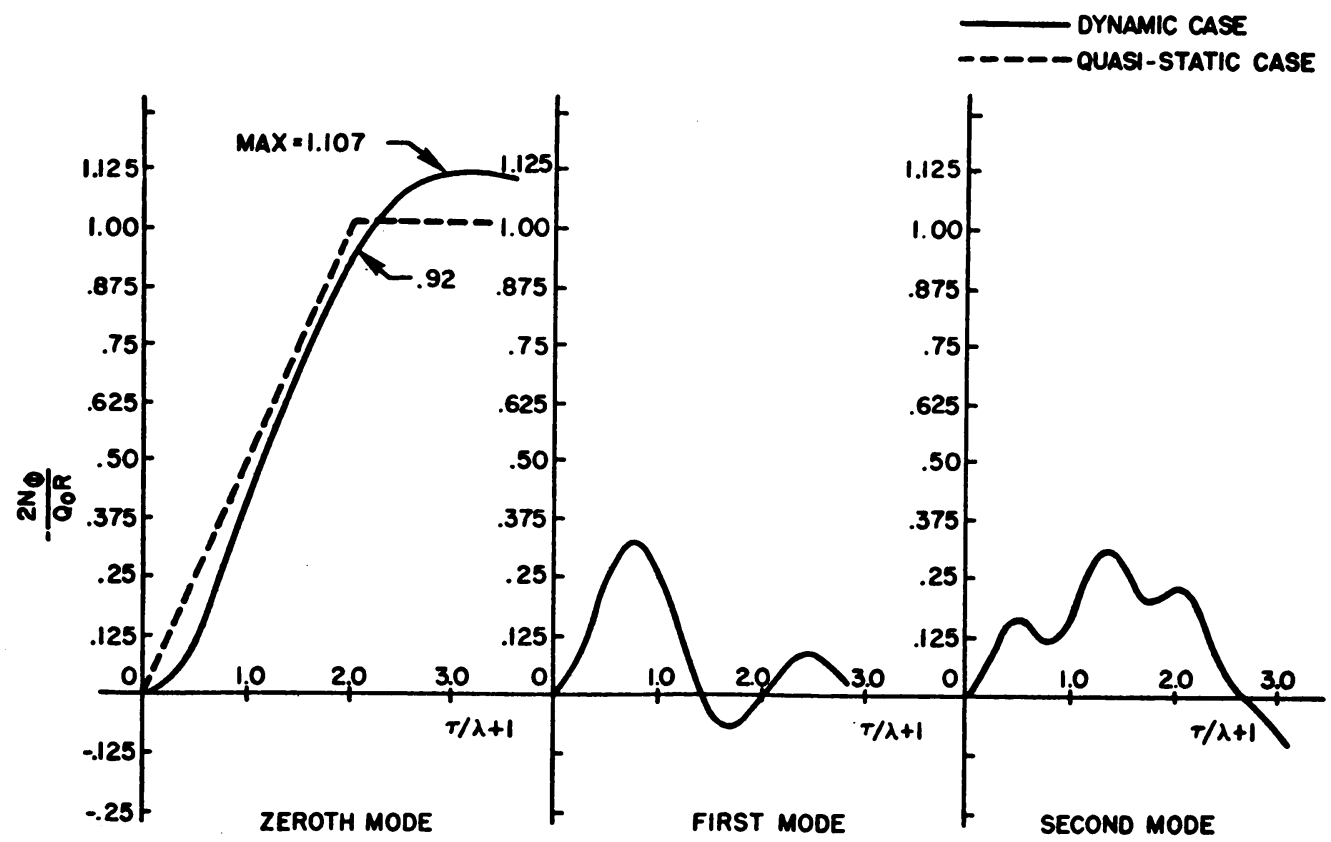

Fig. 2. Hoop stress at $\theta=\pi$ for the first 3 modes.

The parameters were taken as

$$
\beta=17, \quad \delta=0, \quad \lambda^{2}=25 / 3, \quad \nu=.3,
$$

the values appropriate to a step wave of infinite length impinging on a steel shell in water.

From Fig. 2 it becomes evident that the series representing the stresses in the shell converge rapidly enough so that a very good approximation to the total stress may be obtained by considering just the first few modes. Of these, the zeroth mode, which has the steady state solution as its limiting form, is the most important. In this connection it should be noted that the curves in Fig. 2, which were made for $\theta=\pi$, show the largest stresses which can occur in the first and second modes at any time. The zeroth mode is, of course, $\theta$ - independent.

The computation of the acceleration is not so simple. A picture of the total acceleration cannot be gained by looking at the lower modes. In fact, it would seem from Fig. 3 that a few terms of the series $\sum_{n=0}^{\infty} \partial^{2} w_{n} / \partial \tau^{2} P_{n}(\cos \theta)$ will not approximate with acceptable accuracy the total acceleration for all $\tau$ and $\theta$. The rate of change of radial acceleration is very great at $\tau / \lambda=-1, \theta=\pi$; probably the total radial acceleration will be discontinuous at $\tau / \lambda=-1, \theta=\pi$. That this is actually so can be seen by recalling that the incoming wave reaches the point $(1, \theta)$ on the sphere at time $\tau / \lambda=\cos \theta$. In particular, the point of initial impact, $\theta=\pi$, is reached at $\tau / \lambda=-1$. This means that the pressure and, therefore, the radial acceleration as well are discontinuous and that the series representing them do not converge uniformly for $\tau / \lambda=\cos \theta$. The solutions 


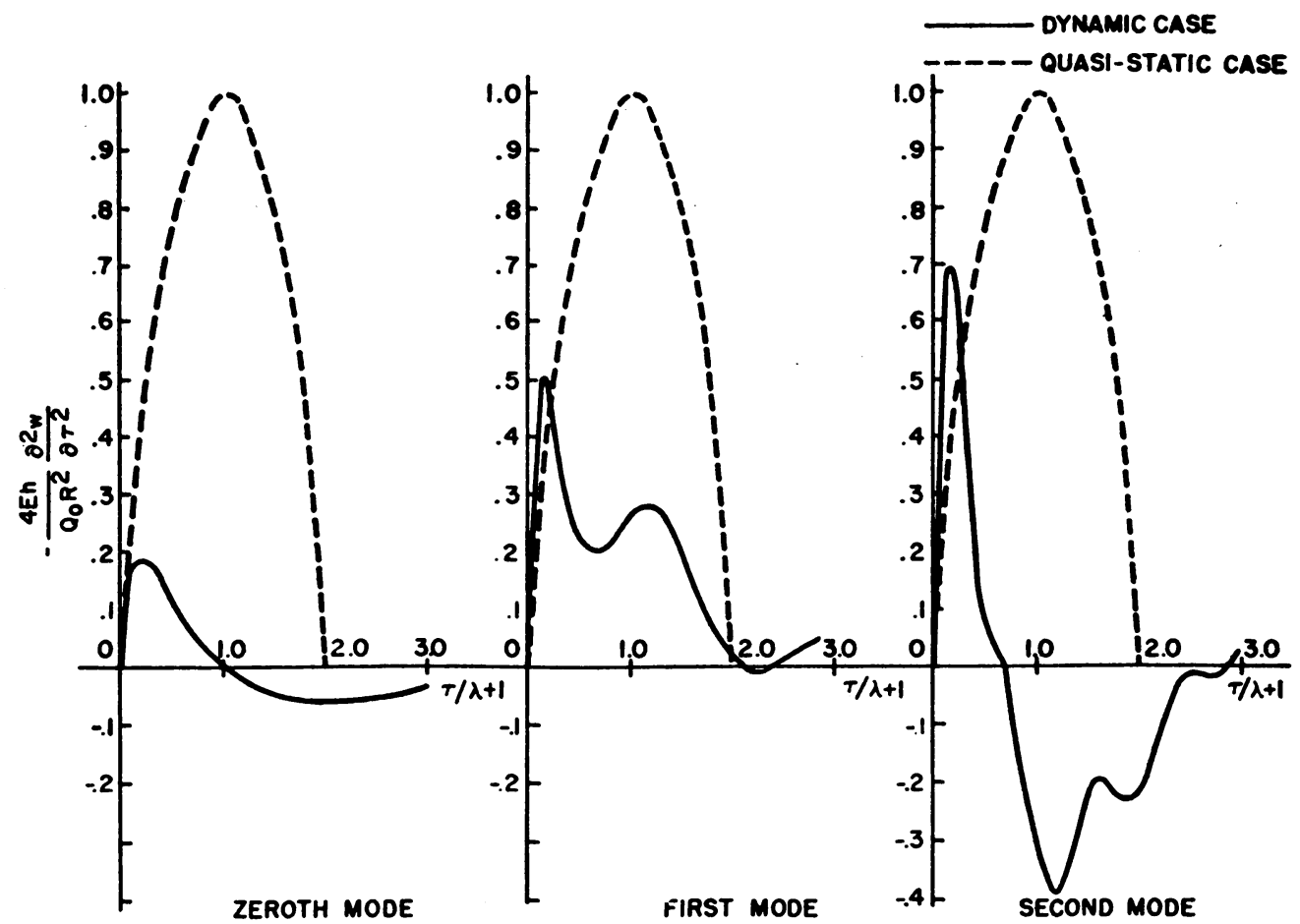

Fra. 3. Radial acceleration at $\theta=\pi$ for the first 3 modes.

for these points must be found in closed form, i.e. when the transform, $\xi^{2} W^{T}(4.17)$, is inverted, the order of summation and integration cannot be interchanged. This difficulty, associated with the use of the series expansion as a method of solution, will be encountered again in Sec. 6 when the resultant pressure distribution is discussed. By anticipating the results of that section, we can find the initial value of $\partial^{2} w / \partial \tau^{2}$ at $\theta=\pi$.

Equation (2.2) gives, for $\tau / \lambda=-1$

$$
\begin{gathered}
s=\rho h \frac{\partial^{2} w}{\partial t^{2}}=\frac{E h}{R^{2}} \frac{\partial^{2} w}{\partial \tau^{2}}, \\
\frac{-4 s}{Q_{0}}=\frac{-4 E h}{Q_{0} R^{2}} \frac{\partial^{2} w}{\partial \tau^{2}} .
\end{gathered}
$$

Taking

$$
s=-2 Q_{0}[(6.6), \theta=\pi]
$$

we have

$$
\frac{-4 E h}{Q_{0} R^{2}} \frac{\partial^{2} w}{\partial \tau^{2}}=8
$$

Thus, while we can obtain a very good approximation to the stresses by considering only the first few modes or even the lowest mode by itself, the same is definitely not true of the acceleration. The actual initial acceleration is about 40 times as great as the maximum acceleration in the zeroth mode. The agreement obtained by considering the 
first and second modes along with the zeroth is not appreciably better; the results still differ by more than a factor of 6 .

Quasi-Static Case. It is of interest to compare the hoop stresses for the zeroth mode with those for the quasi-static case; i.e. for the case in which both scattering and the inertia forces due to deformation are neglected.

Since the effects of only the incident wave are considered, the pressure at time $t$ is given by

$$
\text { pressure }= \begin{cases}\frac{Q_{0} 2 \pi R(t c+R)}{4 \pi R^{2}}=\frac{Q_{0}[(\tau / \lambda)+1]}{2} & -1 \leq \tau / \lambda \leq 1 \\ Q_{0} & 1 \leq \tau / \lambda .\end{cases}
$$

The stresses are given by

$$
N_{\theta}=N_{\phi}= \begin{cases}\frac{-R Q_{0}[(\tau / \lambda)+1]}{4} & -1 \leq \tau / \lambda \leq 1 \\ -R Q_{0} / 2 & 1 \leq \tau / \lambda .\end{cases}
$$

$-2 N_{s} /\left(R Q_{0}\right)$ appears as the dotted line in Fig. 2, where it can be seen that the very simple quasi-static case approximates the more exact dynamic case very closely. The stress developments, while slightly out of phase, are essentially parallel with a difference in maxima of only 10 per cent.

For $-1 \leq \tau / \lambda<1$, there will be an unbalanced force, due to the incident wave, acting on the sphere. This will result in a rigid-body acceleration in the $z$ direction. The magnitude of the force is

$$
Q_{0} \pi R^{2}\left[1-(\tau / \lambda)^{2}\right]
$$

therefore

$$
4 \pi R^{2} h \rho \frac{\partial^{2} z}{\partial \tau^{2}}=Q_{0} \pi R^{2}\left[1-(\tau / \lambda)^{2}\right]
$$

or

$$
\frac{4 E h}{Q_{0} R^{2}} \frac{\partial^{2} z}{\partial \tau^{2}}=\left[1-(\tau / \lambda)^{2}\right]
$$

This has been plotted for purposes of comparison in Fig. 3.

The formal resemblance is greatest for the first mode. The maximum acceleration in the zeroth mode is only one fifth the rigid-body acceleration. For higher modes, the maxima move closer to the rigid-body value of 1 . By referring to the previous section, however, we see that the maximum total acceleration associated with deformation is 8 times that of the rigid body.

6. Resultant pressure distribution. From (3.2), (4.1), (4.3), (4.15), and (4.21) and $(4.9 \mathrm{~b})$ the total pressure is known to be 


$$
\begin{aligned}
P_{\text {Total }}= & P_{\mathrm{I}} \text { (incident) }+P_{\mathrm{II}}=-p^{*} \phi_{. \digamma}=-Q_{0} \chi_{. \digamma}, \\
= & Q_{0}-\frac{Q_{0 i}}{2 \pi} \int_{-i \alpha-\infty}^{-i \alpha+\infty} \exp (i \xi \tau) \psi^{T} \xi d \xi, \\
= & Q_{0}+\frac{Q_{0 i}}{2 \pi} \int_{-i \alpha-\infty}^{-i \alpha+\infty} \xi \exp (i \xi \tau) \sum_{n=0}^{\infty} \frac{(-i)^{n}(2 n+1)}{2 \xi^{2}} h_{n}^{(2)}(\lambda \xi r)[1 \\
& \left.\quad+\frac{C_{1} \lambda h_{n}^{(1) \prime}+i C_{2} h_{n}^{(1)}}{C_{1} \lambda h_{n}^{(2)}+i C_{2} h_{n}^{(2)}}\right] P_{n}(\cos \theta) d \xi .
\end{aligned}
$$

Unfortunately, the order of summation and integration in (6.1) cannot be interchanged for all $\theta, \tau$ and $r$; the total pressure cannot be approximated by the sum of the first $n$ vibrational modes. This can be seen for the specific case $r=1, \tau=-\lambda, \theta=\pi$ as follows. At the moment of initial impact, $\tau=-\lambda$, we have for each mode and for all $\theta, N_{0}=N_{0}=\partial^{2} v_{n} / \partial \tau^{2}=\partial^{2} w_{n} / \partial \tau^{2}=0$. The equations of equilibrium, (2.1) and (2.2), require that the pressure at the surface of the sphere must likewise vanish for each mode at $\tau=-\lambda$, so that the total pressure would be zero for all $\theta$. We should expect, however, from what is known of the theory of scattering of plane waves, that the pressure $Q_{0}$ would be doubled and not reduced to zero.

$\psi^{T}$ must be found in closed form if $P_{\text {Total }}$ is to be evaluated. It has not proved feasible to carry out the summation for all $\theta, r$ and $\tau$. However, it was noted that the expansions for $\psi_{0}^{T}$ and $\psi^{T}$ are very similar for $r=1$, and $\xi$ very large or $[(\tau / \lambda)-\cos \theta]$ very small. This fact can be used to obtain the pressure at the surface of the sphere for $\tau / \lambda \approx \cos \theta$.

By referring to the expression for $\psi_{0}^{T}(4.16)$, it is seen that if we set $\lambda \xi=\zeta$, the pressure associated with the incident wave may be written

$$
P_{1}=Q_{0}=-\frac{Q_{0 i}}{2 \pi} \int_{-i \alpha \lambda-\infty}^{-i \alpha \lambda+\infty} \frac{\exp (i \zeta \tau / \lambda)}{\zeta} \sum_{n=0}^{\infty} j_{n}(\zeta r) P_{n}(\cos \theta)(2 n+1)(-i)^{n} d \zeta
$$

For $\zeta \gg \beta$ and $r=1, P_{I I}$, (see 6.1), becomes, to first order

$$
\begin{aligned}
P_{\mathrm{II}}=\frac{Q_{0}}{2 \pi} \int_{-i \alpha \lambda-\infty}^{-i \alpha \lambda+\infty} \frac{\exp (i \zeta \tau / \lambda)}{\zeta} & \sum_{n=0}^{\infty} \\
& \cdot \frac{\sin [\zeta-(n \pi / 2)-\pi / 2]}{\zeta}(2 n+1)(-i)^{n} P_{n}(\cos \theta) d \zeta
\end{aligned}
$$

and $P_{I}$ reduces to

$$
\begin{aligned}
P_{\mathrm{I}}=-\frac{Q_{0 i}}{2 \pi} \int_{-i \alpha \lambda-\infty}^{-i \alpha \lambda+\infty} \frac{\exp (i \zeta \tau / \lambda)}{\zeta} \sum_{n=0}^{\infty} \\
. \frac{\cos [\zeta-(n \pi / 2)-\pi / 2]}{\zeta}(2 n+1)(-i)^{n} P_{n}(\cos \theta) d \zeta .
\end{aligned}
$$

Equations (6.3) and (4.14) tell us that

$$
\zeta \exp (-i \zeta \cos \theta) \underset{\zeta \rightarrow \infty}{\rightarrow} \sum_{n=0}^{\infty} \cos [\zeta-(n \pi / 2)-\pi / 2](2 n+1)(-i)^{n} P_{n}(\cos \theta)
$$


so that to first order:

$$
\begin{array}{rlrl}
P_{\mathrm{II}} & =\frac{Q_{0}}{2 \pi} \int_{-i \alpha \lambda-\infty}^{-i \alpha \lambda+\infty} \frac{\exp \{i \zeta[(\tau / \lambda)-\cos \theta]\}}{\zeta} \exp [i(\pi / 2) \cos \theta] d \zeta, \\
& =Q_{0} \exp [i(\pi / 2)(\cos \theta+1)] \quad \text { for } 0 \leq[(\tau / \lambda)-\cos \theta] \ll .05, \\
& =0 & \text { for }[(\tau / \lambda)-\cos \theta]<0 .
\end{array}
$$

Higher order terms may be obtained in the same way. To second order:

$$
\begin{aligned}
& P_{\mathrm{II}}=\frac{Q_{0}}{2 \pi} \int_{-i \alpha \lambda-\infty}^{-i \alpha \lambda+\infty} \frac{\exp \{i \zeta[(\tau / \lambda)-\cos \theta]\}}{\zeta}\left\{\exp [i(\pi / 2) \cos \theta]+\left[\frac{\beta+1}{\zeta}\right][1\right. \\
&\left.\quad+i \exp (i(\pi / 2) \cos \theta)]-\frac{\pi}{2 \zeta} \exp [i(\pi / 2) \cos \theta]\right\} d \zeta, \\
&= Q_{0}\{\exp [i(\pi / 2) \cos \theta+i \pi / 2]-[(\tau / \lambda)-\cos \theta][1+\beta][1 \\
&\quad+\exp (i(\pi / 2) \cos \theta+i \pi / 2)]+\pi / 2[(\tau / \lambda)-\cos \theta][\exp (i(\pi / 2) \cos \theta)]\} \\
&=0 \text { for } 0 \leq[(\tau / \lambda)-\cos \theta] \ll .05,
\end{aligned}
$$

Additional terms will be of little value since the representation is valid only for $\zeta \gg \beta,[(\tau / \lambda)-\cos \theta] \ll .05$. The terms found so far, however, are sufficient to tell us some things of importance.

The incoming wave will reach the point $(1, \theta)$ on the sphere at time $\tau / \lambda=\cos \theta$. The pressure on impact for each $\theta$ is given by ${ }^{4}$

$$
P_{\text {Total }}=P_{1}+\operatorname{Re} P_{11}=Q_{0}+Q_{0} \cos [(\pi / 2)(\cos \theta+1)] .
$$

At $\theta=\pi$, the outermost point of the sphere, the first effect is that of a plane wave hitting a rigid wall and we have

$$
P_{\text {II }}=P_{\text {I }} ; \quad P_{\text {Total }}=2 Q_{0} .
$$

At $\theta=\pi / 2$, the wave just grazes the sphere and therefore

$$
P_{\text {II }}=0 ; \quad P_{\text {Total }}=Q_{0} .
$$

As $\theta$ varies from $\pi$ to $\pi / 2$, the initial pressure varies continuously from $2 Q_{0}$ to $Q_{0}$. If the sphere were rigid, the steady-state pressure distribution would be given by

$$
P_{\text {Total }}=P_{\mathrm{I}} \quad 0 \leq \theta \leq \pi .
$$

The results of Sec. 5 indicate that we will have asymptotic values $R Q_{0} / 2$ for the stresses and zero for the radial acceleration, which also correspond to a uniform pressure of $Q_{0}=P_{I}$.

The elastic waves in the shell will travel more rapidly than the acoustic wave and will result in a pressure, $P \neq 0$, at $(1, \theta)$ before the time $\tau / \lambda=\cos \theta, \theta \neq \pi$. However, this effect is negligibly small compared with the one we are considering. 
At present, this is about all that can be said on the subject of the pressure distribution. While the series method is very convenient for finding the stresses and may be adapted to give results for the radial acceleration, it does not lend itself to an analysis of the pressure distribution. It would seem that, for this aspect of the problem, another approach is called for.

7. Remarks. In a paper which appeared after this work was completed, J. H. Huth and J. D. Cole [6] consider the related problem of the effect of a shock wave on an airborne elastic spherical shell.

This problem is more difficult than the one treated here, and the authors have introduced the simplifying assumption that the effect of the diffracted wave on the shell may be neglected, or, in other words, that the effect on the applied pressure of both the motion of the sphere and the sphere itself may be neglected.

The stresses, but not the radial acceleration, are computed. The problem of finding the resultant pressure distribution does not, of course, arise.

It would be of interest to compare their results for the limiting case of a very weak shock (i.e. speed of shock wave $\rightarrow$ speed of sound) with the stresses that would be obtained here by using for $\beta$ and $\lambda$ (see Sec. 3) the values appropriate to a steel shell in air. Such computations are now under way.

\section{BibliograpHY}

1. G. F. Carrier, The interaction of an acoustic wave and an elastic cylindrical shell, Brown University report to the Office of Naval Research under Contract N7onr-35810, No. B11-4, 1951

2. R. D. Mindlin and H. H. Bleich, Response of an elastic cylindrical shell to a transverse, step shock wave, J. Appl. Mech. 20, No. 2, 189-195 (1953)

3. J. H. Jellet, On the properties of extensible surfaces, Roy. Irish Acad. Trans. 22, Part V (1855)

4. S. Timoshenko, Theory of plates and shells, 1st Ed., McGraw-Hill, New York and London, 1940

5. H. Lamb, On the vibrations of a spherical shell, Proc. London Math Soc. 14, 50 (1883)

6. J. H. Huth and J. D. Cole, Elastic stress waves produced by pressure loads on a spherical shell, J. Appl. Mech. 22, No. 4, 473-478 (1955) 